Rock Mech. Rock Engng. (2007) 40 (2), 193-211

DOI 10.1007/s00603-006-0095-6

\title{
Application of Dimensional Analysis in Calibration of a Discrete Element Model for Rock Deformation and Fracture
}

\author{
By \\ A. Fakhimi ${ }^{1,2}$ and T. Villegas ${ }^{3}$ \\ ${ }^{1}$ Departments of Mineral and Mechanical Engineering, New Mexico Tech, \\ Socorro, NM, U.S.A. \\ ${ }^{2}$ Department of Civil Engineering, University of Tarbiat Modarres, \\ Tehran, Iran \\ ${ }^{3}$ Department of Civil and Mining Engineering, University of Sonora, \\ Hermosillo, Sonora, Mexico
}

Received January 15, 2005; accepted March 18, 2006

Published online June 9, 2006 (C) Springer-Verlag 2006

\begin{abstract}
Summary
A discrete element approach was used in the simulation of rock fracture. The numerical synthetic material was made of rigid circular particles or cylinders that have interaction through normal and shear springs. The cylinders were bonded to each other at the contact points to withstand the applied loads. To characterize the microscopic properties of this synthetic material, a dimensional analysis approach was presented. It was shown that the dimensionless parameters and graphs obtained were useful tools for fast and efficient calibration of a synthetic material. This calibration method was employed for finding a numerical model for Pennsylvania Blue Sandstone. The numerical model could mimic many deformational and failure characteristics of the sandstone in both conventional and some non-conventional stress paths.
\end{abstract}

Keywords: Rock fracture, discrete element method, dimensional analysis, calibration.

\section{List of Symbols}

D specimen width and specimen diameter

E Young's modulus

$\mathrm{E}^{\prime} \quad$ apparent Young's modulus

$k_{n} \quad$ normal stiffness of a contact

$k_{s} \quad$ shear stiffness of a contact

$n_{b} \quad$ normal bond of a contact

$p \quad$ maximum applied load in a Brazilian test and average of maximum and minimum principal stresses

$q \quad$ half of difference between maximum and minimum principal stresses 


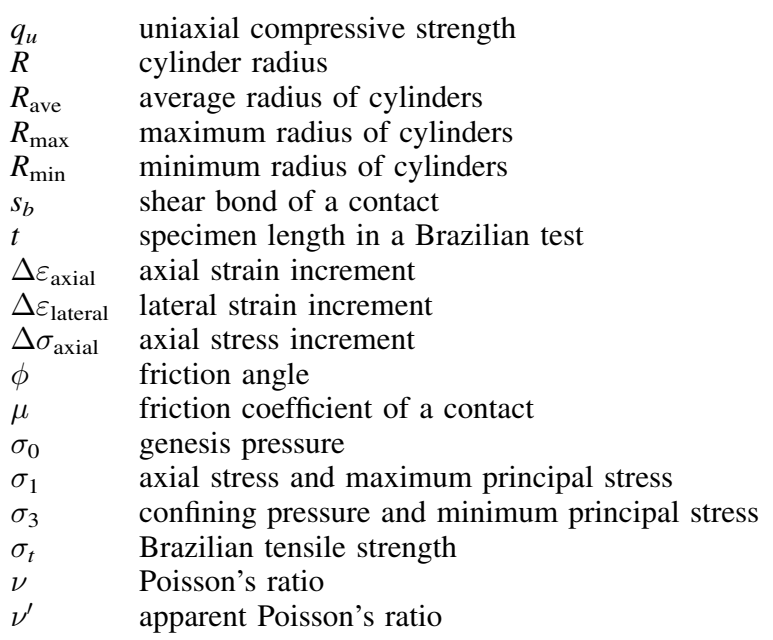

\section{Introduction}

The issue of rock deformation and fracture has been studied intensively by researchers in the past. Two general paths are usually followed to investigate rock fracture, a continuum model and a discrete model. In the continuum model, different techniques are implemented; the most common ones are softening plasticity and damage mechanics models. In the softening approach, rock friction angle or cohesion can be considered to evolve by an invariant of plastic strain or plastic work (Vermeer and De Borst, 1984), while in the damage model, rock stiffness is reduced with deformation (De Borst, 2002). In the continuum framework, the geomaterial failure and localization of deformation can be studied as a bifurcation phenomenon as well (Rudnicki and Rice, 1975; Vardoulakis and Sulem, 1995).

Crack propagation in rock features a complex behavior that usually needs to be studied numerically. The difficulty with the numerical rock softening and damage analysis is the dependency of the failure load on the mesh resolution. For example, it is well known that in the finite element analysis of rock softening, by reducing the mesh size, the dissipated energy in the fracture zone or shear band reduces which is not physically meaningful (Bazant and Planas, 1998). To remedy this difficulty, nonlocal continuum (Bazant and Pijaudier-Cabot, 1988), Cosserat continuum (De Borst and Muhlhaus, 1991) and gradient plasticity techniques (De Borst and Muhlhaus, 1992) have been proposed in the literature.

Numerical continuum methods based on micromechanics and flaw distribution have been proposed as well. In a multi-laminate model (Pande and Xiong, 1982), discrete failure planes are considered in otherwise a continuum system. The original multi-laminate model later on was renamed a microplane model in that a geometric damage tensor was introduced (Carol et al., 1991). In a microplane model, a non-local averaging technique needs to be implemented to avoid the sensitivity of the results to mesh resolution. A displacement discontinuity technique was used by Van de Steen et al. (2003) to model rock fracture. This technique was successfully implemented in simulating rock spalling around an underground excavation, although no comment 
was presented regarding calibration of the model. Fractures can be studied as mathematical cracks as well. In this case, linear fracture mechanics or cohesive crack models (Bazant and Planas, 1998) can be used, although extension of these models to biaxial compressive loading is not straightforward.

In the discrete element approach of rock fracture, the interaction of particles is considered explicitly in the model. This provides an embedded internal length in the formulation of the problem that prevents the numerical difficulties observed in the classical local continuum softening models. The application of this technique in geomechanics was initially proposed by Cundall and Strack (1979) and later on extended and implemented in the PFC computer programs (Itasca, 1999). With this technique, crack propagation, rock softening and dilation, anisotropic rock behavior in damaged zone, cyclic energy dissipation, spontaneous emission of acoustic energy and Coulomb failure envelope can be reproduced. Nevertheless, this powerful technique has a few shortcomings. Firstly, it needs calibration, i.e. some micro-mechanical properties must be specified to result in a synthetic material with desired macro-mechanical properties such as Young's modulus, Poisson's ratio, tensile strength, and failure envelope. For a simple contact bond model implemented in this paper, the micro-mechanical properties for interaction of two circular particles or cylinders are normal and shear spring stiffnesses $\left(k_{n}, k_{s}\right)$, normal and shear bonds $\left(n_{b}, s_{b}\right)$ to glue the particles in contact and Coulomb friction coefficient $(\mu)$ which is activated when the bond between any two particles is broken. Therefore, these five parameters must be obtained during the calibration step. The calibration can be performed by using a trial and error approach in that extensive effort is needed to acquire appropriate micro-parameters to result in macroscopic properties similar to those of a specific rock. A more efficient method was employed by Huang (1999) for calibration of a circular particle interaction (CPI) model in that a dimensional approach was established and some dimensionless parameters were introduced.

The second problem with a CPI model is the difficulty of calibrating it for the failure envelope. In general, a CPI model shows a friction angle which is smaller than that for a hard rock (Potyondy and Cundall, 2001). To increase the friction angle of a particle assemblage, non-circular particles can be used (Ting et al., 1993), but this requires additional computational time to detect contact of adjacent particles; contact detection of circular particles is much faster compared to that of other particle shapes.

The third difficulty of a CPI model is ratio of unconfined compressive strength to tensile strength which is usually lower than that of a rock. Fakhimi (2004) proposed a slightly overlapped circular particle interaction (SOCPI) model to resolve the above second and third problems. The calibration issue of a SOCPI material that is based on the dimensional analysis is addressed in this paper. The calibration graphs introduced were used to develop a synthetic model for Pennsylvania Blue Sandstone. Several physical and numerical tests are reported to verify the validity of the numerical model.

All the numerical analyses in this paper were conducted using CA2 computer program which was developed by the first author (Fakhimi, 1998). CA2 is a hybrid finite difference-discrete element code for large deformation static or dynamic deformation of solid materials. The first step in discrete element modeling of rock, using $\mathrm{CA} 2$, is sample preparation. This step involves with generation of walls that define the domain of analysis. The walls in CA2 are not rigid but are discretized to smaller elements and are used to confine the cylinders. Next, the cylinders with uniformly 
distributed radii are generated and inflated by increasing the cylinders radii. An explicit algorithm is used to obtain the equilibrium configuration of this particle assembly. At this stage of analysis, a contact bond model with no friction and bond at the contact points is assumed for the interaction of cylinders. After equilibrium, the induced stress in the surrounding walls is examined. By changing the number of cylinders, the induced stress can be modified. This stress is called genesis pressure $\left(\sigma_{0}\right)$ and its role is to create a small overlap between cylinders in contact. This small overlap is responsible for obtaining more realistic friction angle and ratio of compressive to tensile strength of the synthetic material (Fakhimi, 2004). The last step in sample preparation is to initialize the normal and shear contact stresses to zero and to introduce normal and shear bonds and friction between the cylinders in contact. More details about sample preparation are given in Fakhimi (2004).

\section{Calibration Charts}

Calibration of a synthetic model made of rigid circular cylinders is a tedious task if it is undertaken by using a trial and error approach. A much more convenient and efficient method is to introduce some calibration curves using a dimensional analysis. In this paper, a synthetic material was intended to be calibrated for Young's modulus, Poisson's ratio, failure envelope and tensile strength. A SOCPI model is calibrated by assigning appropriate parameters including normal and shear spring constants $\left(k_{n}, k_{s}\right)$, normal and shear bonds $\left(n_{b}, s_{b}\right)$, friction coefficient $(\mu)$, genesis pressure $\left(\sigma_{0}\right)$, average cylinders radius $(R)$ and specimen width $(D)$.

The parameters that dictate the elastic properties of a SOCPI material are $k_{n}, k_{s}, \sigma_{0}$, $R$ and $D$. These five parameters have two independent dimensions, i.e. force $(F)$ and length $(L)$. Hence, considering the p theorem in dimensional analysis (Sedov, 1993), $5-2=3$ dimensionless parameters are involved in describing the elastic deformation of the synthetic material, i.e.

$$
\begin{gathered}
E^{\prime}=k_{n} f\left(R / D, k_{s} / k_{n}, \sigma_{0} / k_{n}\right), \\
\nu^{\prime}=g\left(R / D, k_{s} / k_{n}, \sigma_{0} / k_{n}\right),
\end{gathered}
$$

where $f$ and $g$ are dimensionless functions and $\mathrm{E}^{\prime}$ and $\nu^{\prime}$ are apparent Young's modulus and Poisson's ratio of the synthetic material, defined in a uniaxial test as:

$$
\begin{gathered}
E^{\prime}=\Delta \sigma_{\text {axial }} / \Delta \varepsilon_{\text {axial }} \\
\nu^{\prime}=-\Delta \varepsilon_{\text {lateral }} / \Delta \varepsilon_{\text {axial }} .
\end{gathered}
$$

If a numerical experiment is considered as a biaxial plane strain test, true Young's modulus and Poisson's ratio of synthetic material can be obtained from the following equations:

$$
\begin{aligned}
& \nu=\nu^{\prime} /\left(1+\nu^{\prime}\right), \\
& E=E^{\prime}\left(1-\nu^{2}\right) .
\end{aligned}
$$

Several numerical uniaxial tests were conducted on the synthetic material with $R / D=0.013$ and different $k_{s} / k_{n}$ and $\sigma_{0} / k_{n}$ values, using CA2 computer program. 


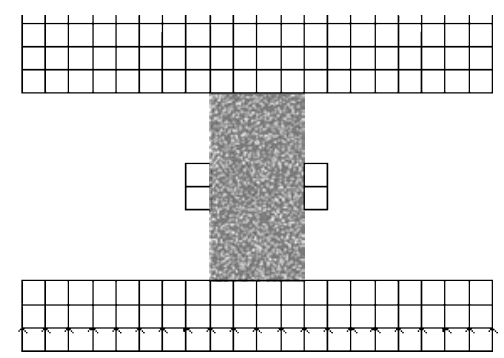

Fig. 1. Numerical unconfined compressive test set-up

The numerical uniaxial test set up together with the synthetic specimen is shown in Fig. 1. The upper and lower platens or walls are made of finite difference grid and are frictionless. The upper wall is fixed vertically while the lower one was moved with a constant velocity to compress the specimen. Two small and very flexible walls were glued to the lateral sides of the specimen. These walls were like measurement devices to ac-
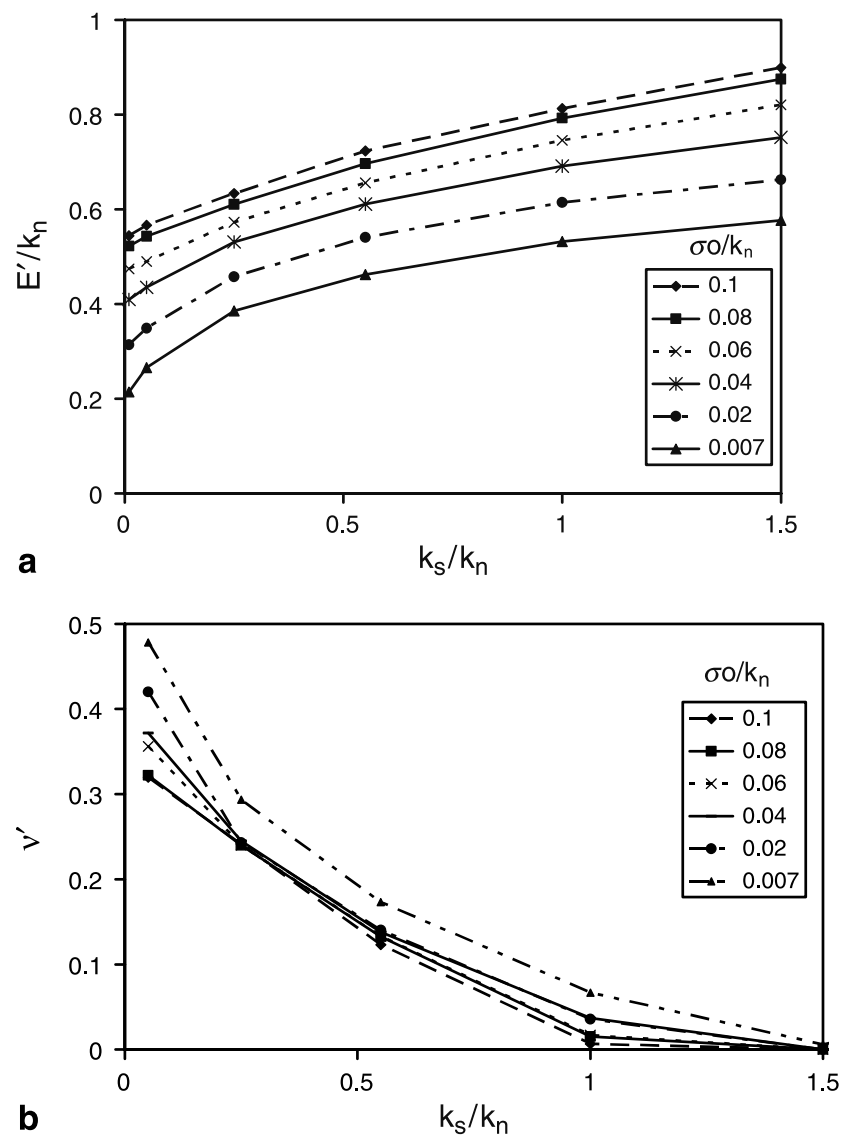

Fig. 2. Elastic constants of synthetic material versus $k_{s} / k_{n}$ for different $\sigma_{0} / k_{n}$, a normalized apparent Young's modulus, b apparent Poisson's ratio 
quire average lateral deformation of the middle of the specimen and consequently the Poisson's ratio. The results of the numerical analyses are shown in Fig. 2a and b. Parametric studies and also the work by Potyondy and Cundall (2001) show that $\mathrm{E}^{\prime}$ and $\nu^{\prime}$ are almost independent of $R / D$ values provided that this ratio is kept small enough. Therefore, by introducing the macroscopic parameters $\mathrm{E}^{\prime}$ and $\nu^{\prime}$ and assuming a value for $\sigma_{0} / k_{n}$, normal and shear spring constants can be estimated from Fig. 2a and b.

The uniaxial strength of the synthetic material $\left(q_{u}\right)$ was obtained by conducting several numerical tests. This parameter is a function of $\sigma_{0}, k_{n}, k_{s}, R, D, n_{b}, s_{b}$, and $\mu$. These eight parameters have two dimensions. Hence, dimensionless unconfined compressive strength can be expressed as:

$$
q_{u} R / n_{b}=f_{1}\left(\sigma_{0} / k_{n}, k_{s} / k_{n}, k_{n} R / n_{b}, n_{b} / s_{b}, R / D, \mu\right),
$$

where $f_{1}$ is a dimensionless function. Numerical analyses demonstrate that $q_{u}$ is a weak function of $\mu$ and $k_{n} R / n_{b}$ (Villegas, 2004). In addition, it has been shown that the biaxial compressive strength of a synthetic material made of circular cylinders is not a function of $R / D$ value, provided that $R / D$ is kept a small number (Potyondy and
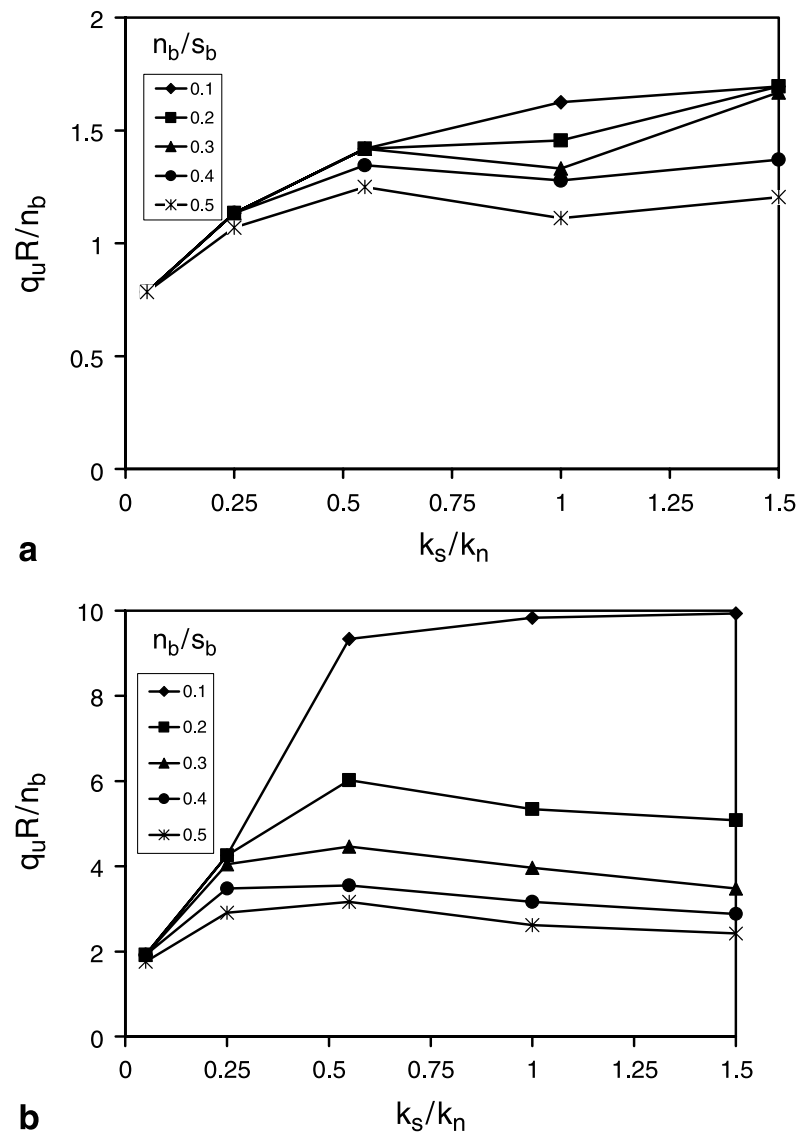

Fig. 3. Dimensionless uniaxial strength versus $k_{s} / k_{n}$ for $\mathbf{a} \sigma_{0} / k_{n}=0.007$ and $\mathbf{b} \sigma_{0} / k_{n}=0.08$ 
Cundall, 2001). Thus, only three dimensionless parameters namely, $\sigma_{0} / k_{n}, k_{s} / k_{n}$ and $n_{b} / s_{b}$ were needed to consider. By conducting uniaxial tests on the synthetic materials with $R / D=0.013\left(R_{\min }=0.44 \mathrm{~mm}\right.$ and $R_{\max }=0.58 \mathrm{~mm}$ with a uniform random distribution of cylinders radii), $\mu=0.5, k_{n}=50 \mathrm{GPa}$, and $n_{b}=20,000 \mathrm{~N} / \mathrm{m}$, two charts, each for a specific value of $\sigma_{0} / k_{n}(0.007$ and 0.08$)$ were obtained. Figure $3 \mathrm{a}$ and $\mathrm{b}$ show these charts where the dimensionless uniaxial strength is plotted versus $k_{s} / k_{n}$ for different $n_{b} / s_{b}$ values. Similar charts for $\sigma_{0} / k_{n}=0.04$ and 0.1 are reported in Villegas (2004). Comparison of these figures verifies that the dimensionless unconfined compressive strength of synthetic material increases as higher values of genesis pressure is used. This should be expected, as with a higher genesis pressure, the number of contact points per unit area of the specimen increases which results in a stronger specimen. It is interesting to observe the effect of $k_{s} / k_{n}$ parameter on the specimen strength. Figure 3 indicate that by reducing the $k_{s} / k_{n}$ parameter to a value less than 0.5 , the dimensionless strength decreases. The crack pattern, in the synthetic material is also affected by the $k_{s} / k_{n}$ ratio. This is demonstrated by the crack pattern for the synthetic specimens with different $k_{s} / k_{n}$ value (Fig. 4 ). A crack in CA2 is a line perpendicular to the line connecting centers of cylinders with broken bond. It is evident from Fig. 4 that for a small $k_{s} / k_{n}$ value, a diffuse crack pattern is obtained, in contrast to a shear banding when a greater value of $k_{s} / k_{n}$ is used. The reason for this observation is that by increasing $k_{s}$ value, greater microscopic shear stresses and hence shear cracks are developed in the specimen with the consequence of shear banding. In addition, it can be shown that a synthetic specimen with a small $k_{s} / k_{n}$ value, features a substantial amount of hardening in its stress-strain diagram, while a greater value of $k_{s} / k_{n}$, results in a more brittle behavior (Villegas, 2004).

Several numerical biaxial tests were conducted to evaluate failure envelope of synthetic material. Strength of synthetic material $\left(\sigma_{1}\right)$ is a function of $\sigma_{0}, R, D, \mu, k_{s}$,

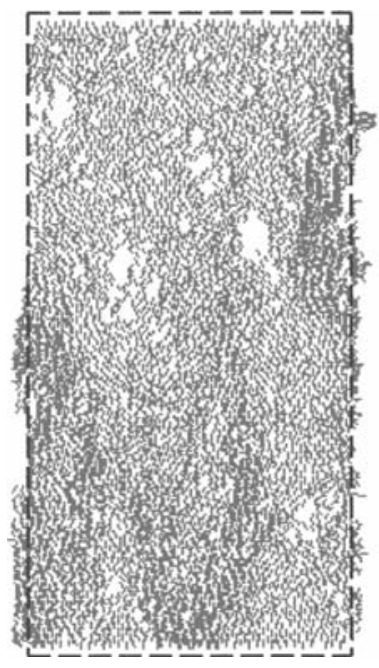

a

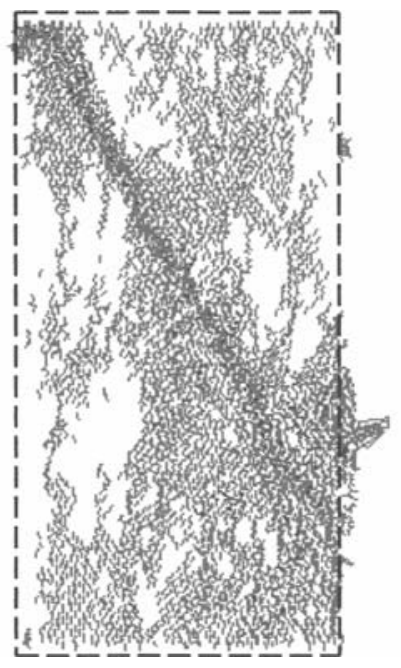

b

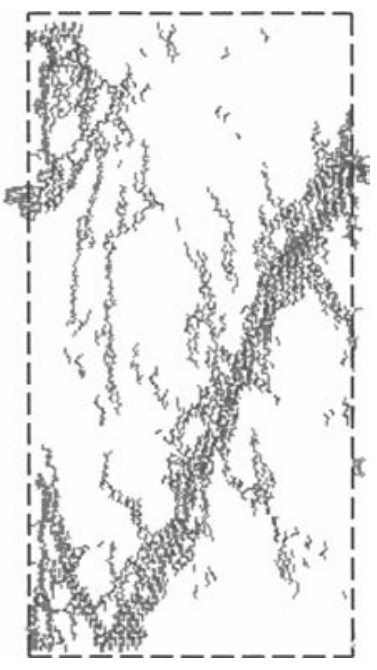

C

Fig. 4. Crack pattern in the damaged specimen with a $k_{s} / k_{n}=0.05, \mathbf{b} k_{s} / k_{n}=0.55$, and $\mathbf{c} k_{s} / k_{n}=1.5$ 
$k_{n}, n_{b}, s_{b}$, and the confining pressure $\left(\sigma_{3}\right)$. Similar to uniaxial strength, $\sigma_{1}$ is a weak function of $\mu$ and $k_{n} R / n_{b}$. Therefore, the biaxial dimensionless strength can be written as:

$$
\sigma_{1} R / n_{b}=g_{1}\left(\sigma_{3} R / n_{b}, k_{s} / k_{n}, \sigma_{0} / k_{n}, n_{b} / s_{b}\right)
$$

where $g_{1}$ is a dimensionless function. The normalized failure envelopes for different values of $\sigma_{0} / k_{n}$ are shown in Fig. 5a-c. In this series of numerical anal-
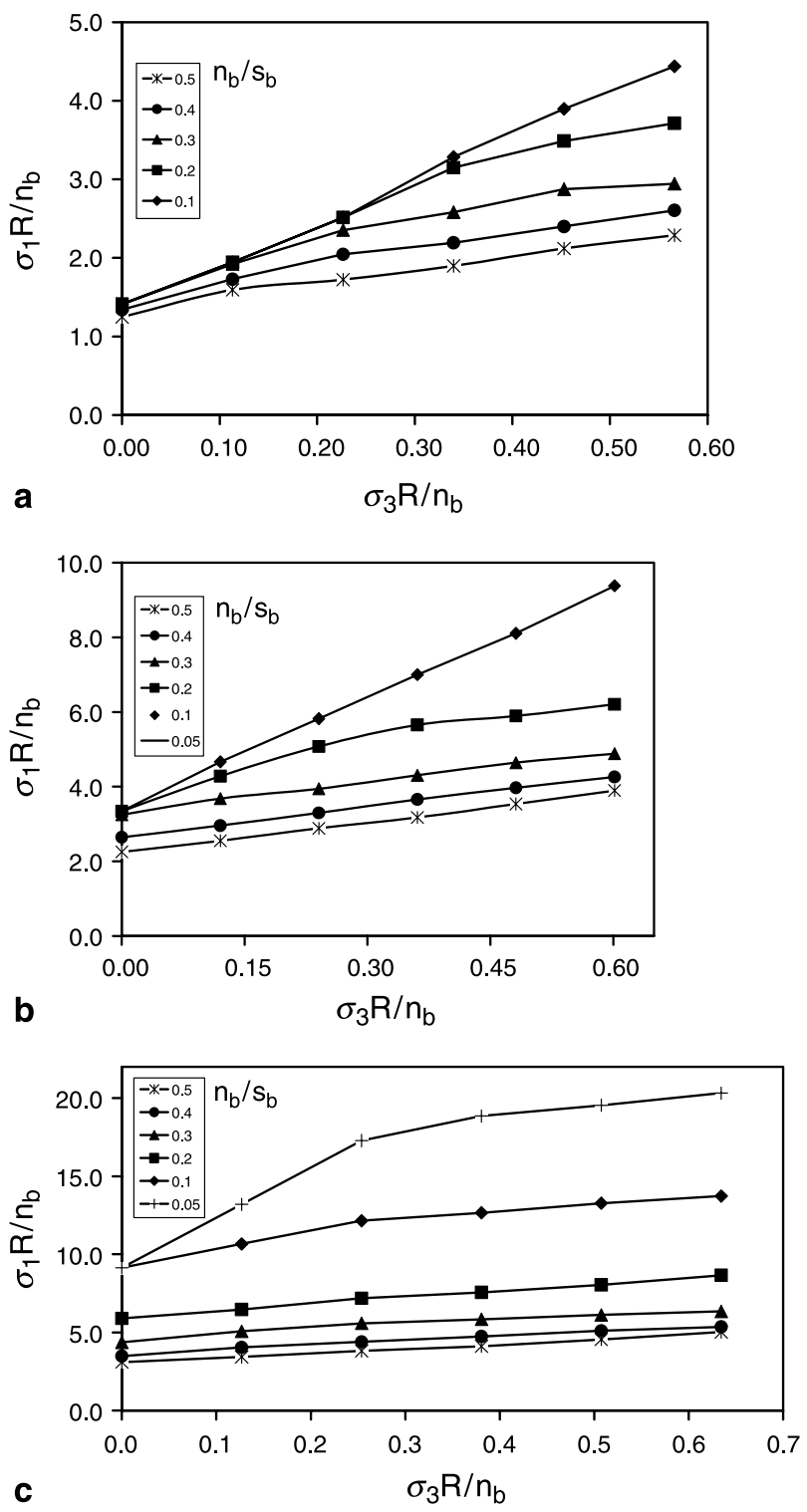

Fig. 5. Normalized failure envelopes for a $\sigma_{0} / k_{n}=0.007$, b $\sigma_{0} / k_{n}=0.04$, and $\mathbf{c} \sigma_{0} / k_{n}=0.08$ 
yses, the following parameters were fixed: $R / D=0.013$ (with $R_{\min }=0.44 \mathrm{~mm}$ and $\left.R_{\max }=0.58 \mathrm{~mm}\right), \mu=0.5, k_{n}=50 \mathrm{GPa}, k_{s}=27.5 \mathrm{GPa}$ and $n_{b}=20,000 \mathrm{~N} / \mathrm{m}$. Different values of $n_{b} / s_{b}(0.05,0.1,0.2,0.3,0.4,0.5)$ were used in the analyses. Figure 5 indicate that by increasing the genesis pressure, specimens with higher strength are obtained. In addition, it is noticed that with larger $\sigma_{0} / k_{n}$ values together with smaller values of $n_{b} / s_{b}$ parameter, curvature of failure envelope similar to that of a rock can be simulated. The slope of failure envelope in a $\sigma_{1}, \sigma_{3}$ space is equal to $\tan ^{2}(45+\phi / 2)$ in that $\phi$ is the friction angle. Inspection of the curves in Fig. 5 for higher $\sigma_{0} / k_{n}$ and smaller $n_{b} / s_{b}$ values show that friction angles of $60^{\circ}$ or more can be simulated. This has become feasible due to the application of non-zero genesis pressure.

To investigate the role of microscopic friction coefficient $(\mu)$ on the failure envelope, two sets of biaxial tests with $\sigma_{0} / k_{n}=0.08, k_{s} / k_{n}=0.55, n_{b} / s_{b}=0.2, k_{n} R / n_{b}=1269$, and $R / D=0.013$ were conducted. For the first set of biaxial tests, a friction coefficient of 0.5 was used while for the second set, $\mu$ was 0.8 . Figure 6 shows the peak and residual failure envelopes for these two synthetic materials. This figure demonstrates that while $\mu$ has a small effect on the peak failure envelope, its role on the residual strength is negligible. Consequently, a great percentage of the induced friction, in the numerical model, is due to the shear displacement along the developed irregular failure surface and to a much less extent the microscopic friction angle $(\mu)$ plays a role.

Some numerical Brazilian tests were performed to evaluate tensile strength of synthetic material. The numerical test set up for the Brazilian test is shown in

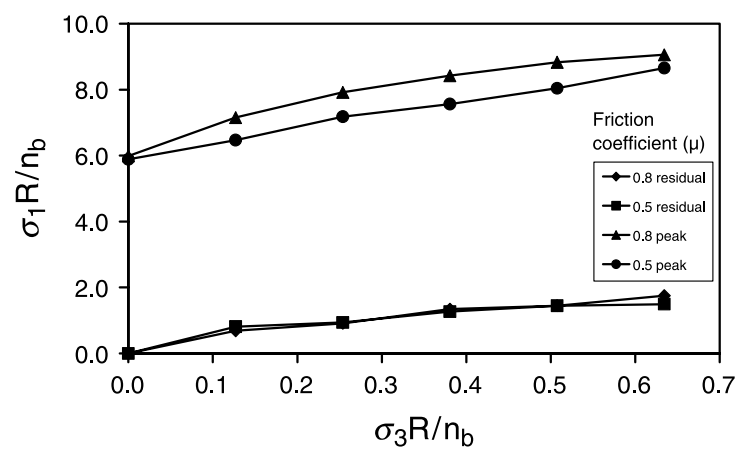

Fig. 6. Normalized peak and residual failure envelopes for two different friction coefficients

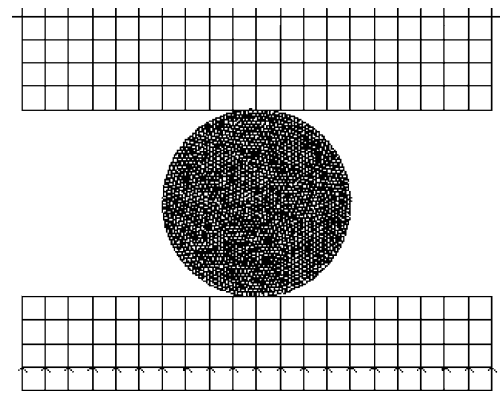

Fig. 7. Numerical Brazilian test set-up 
Fig. 7. The upper platen is fixed in the vertical direction while the lower platen was moved with a constant velocity of $0.2 \times 10^{-8} \mathrm{~m} / \mathrm{step}$ in the upward direction. Both upper and lower platens were discretized to the smaller finite elements. Two series of numerical Brazilian tests were conducted. In the first series, for specific values of $\sigma_{0} / k_{n}$, the tensile strength was obtained by changing $k_{s} / k_{n}$ and $n_{b} / s_{b}$ parameters. The tensile strength was obtained from the following equation (Goodman, 1989):

$$
\sigma_{t}=2 p / \pi D t
$$

where $p$ is the maximum applied load, $D$ is the specimen diameter and $t$ is its length that is equal to unity in the numerical tests. Figure $8 \mathrm{a}$ and $\mathrm{b}$ show the dimensionless tensile strength $\left(\sigma_{t} R / n_{b}\right)$ versus $k_{s} / k_{n}$ parameter for $\sigma_{0} / k_{n}=0.1$ and 0.04 , respectively. Similar to the observation with the unconfined compressive strength, it is evident that increase in genesis pressure results in higher tensile strength. The reason for the observed maximum points of dimensionless tensile strength as shown in Fig. 8a is not clear to the authors. One possible explanation is that by increasing $n_{b} / s_{b}$ parameter with higher values of $\sigma_{0} / k_{n}$, the intensity of micro shear cracks are increased in the model that results in change of the trend of the failure load.
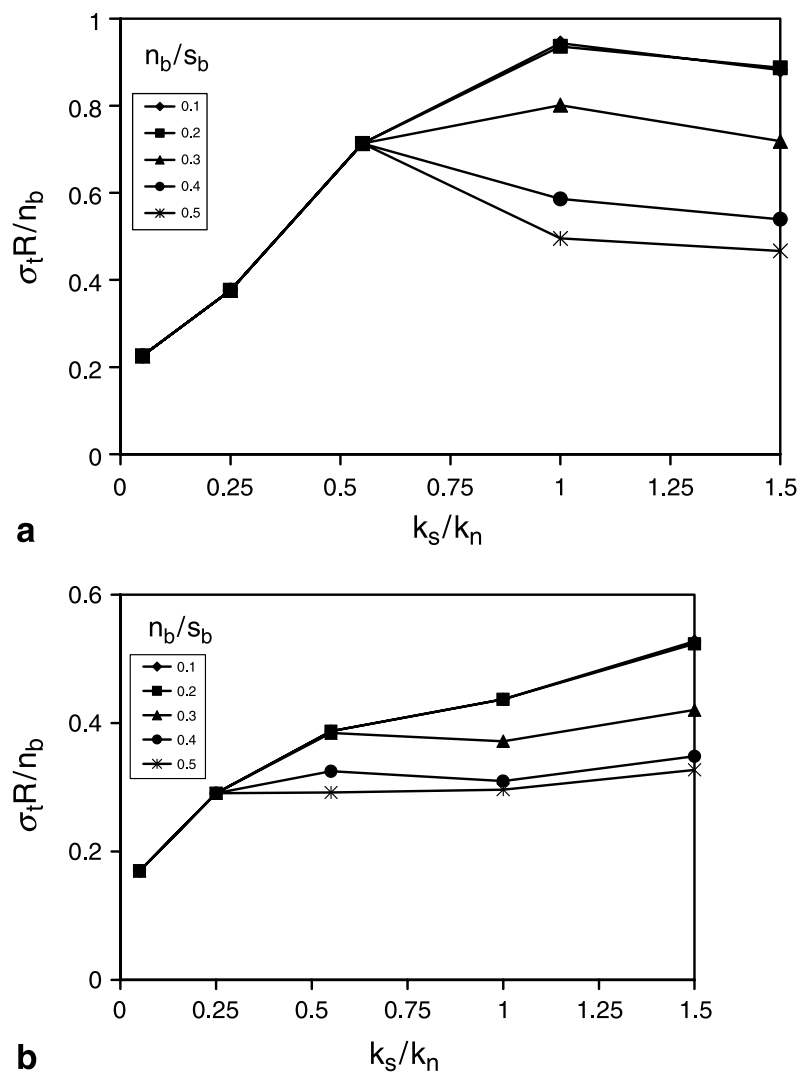

Fig. 8. Dimensionless Brazilian tensile strength versus $k_{s} / k_{n}$ for a $\sigma_{0} / k_{n}=0.1$ and $\mathbf{b} \sigma_{0} / k_{n}=0.04$ 

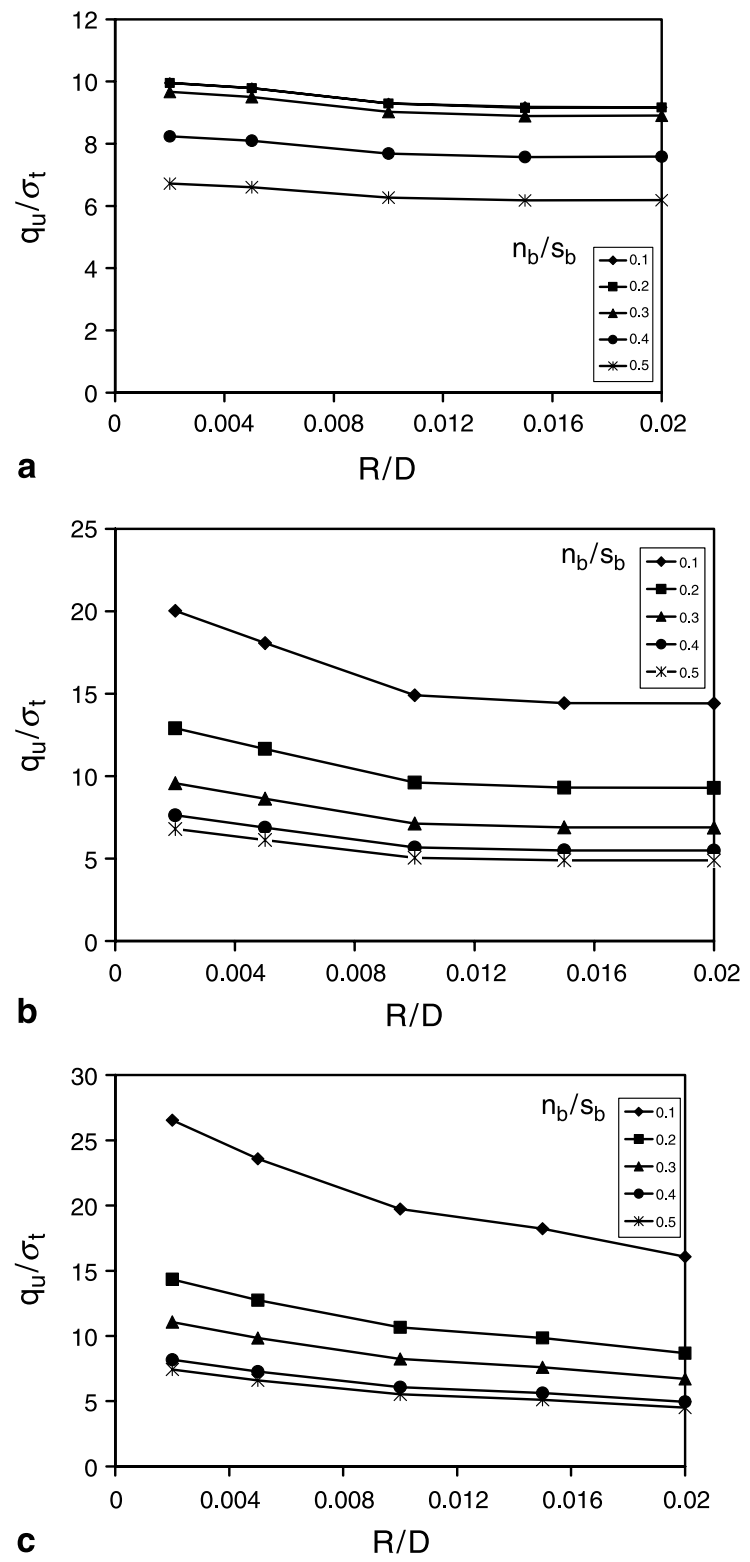

Fig. 9. Ratio of unconfined compressive strength to Brazilian tensile strength versus specimen resolution for a $\sigma_{0} / k_{n}=0.04, \mathbf{b} \sigma_{0} / k_{n}=0.08$, and $\mathbf{c} \sigma_{0} / k_{n}=0.1$

In the second series of Brazilian tests, the size effect was investigated. Figure 9 show the ratio of unconfined compressive strength to tensile strength verses $R / D$ for $\sigma_{0} / k_{n}$ of $0.04,0.08$ and 0.1 . Since unconfined compressive strength appears to be independent of $R / D$, it is realized that $\sigma_{t}$ reduces by decreasing $R / D$ value, for a fixed $n_{b} / R$. This means that for a numerical model with fixed average cylinder radius $(R)$, by 

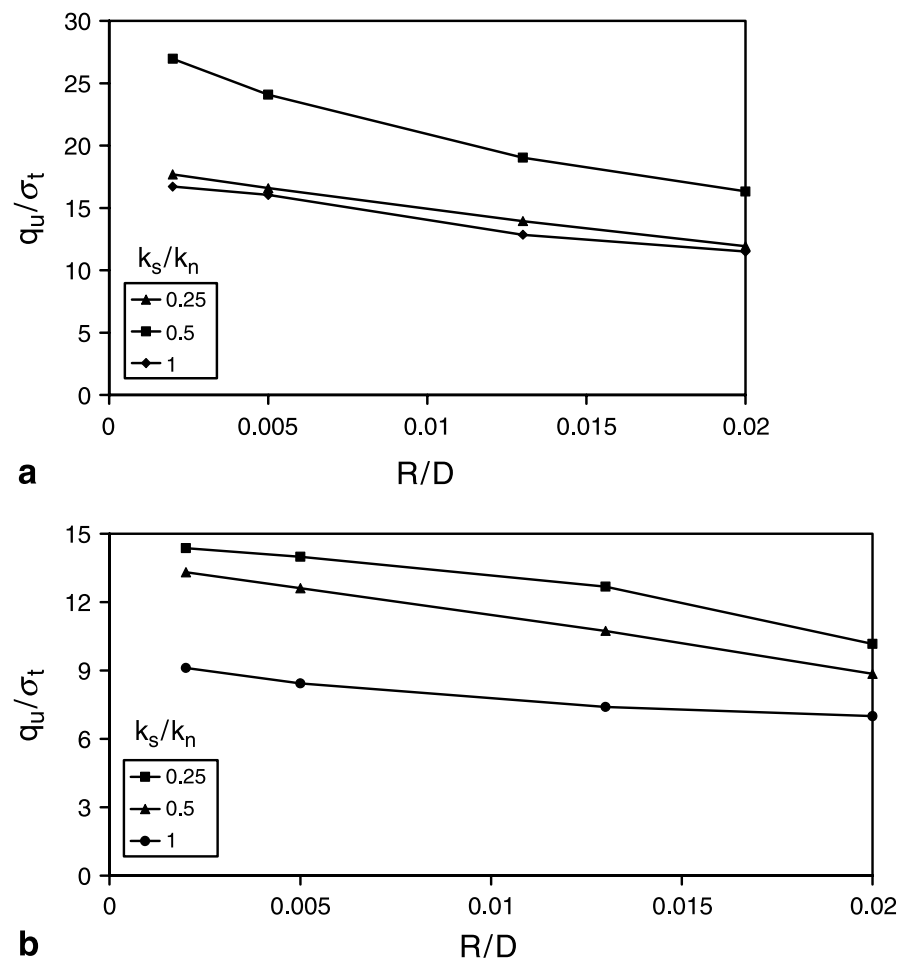

Fig. 10. Ratio of unconfined compressive strength to Brazilian tensile strength versus specimen resolution for different $k_{s} / k_{n}$ values. a $\sigma_{0} / k_{n}=0.1, n_{b} / s_{b}=0.1, \mathbf{b} \sigma_{0} / k_{n}=0.08, n_{b} / s_{b}=0.2$

increasing the specimen size $(D)$, the tensile strength decreases. This size effect has already been reported by Potyondy and Cundall (2001) and Huang (1999) for a CPI model and is confirmed here for a SOCPI material. Notice that specimens with higher genesis pressure and lower $n_{b} / s_{b}$ values demonstrate more intensive size effect. The reason for the observed size effect in a numerical Brazilian test goes back to the dependence of fracture toughness of the synthetic material to the specimen resolution; by increasing the cylinders radii for a fixed $n_{b} / R$, the fracture toughness increases (Potyondy and Cundall, 2001). Figure 10a and b demonstrate $q_{u} / \sigma_{t}$ parameter versus $R / D$ for different $k_{s} / k_{n}$ values. These two graphs are important for the calibration procedure as is shown in this paper. An important observation here is that by introducing a non-zero genesis pressure, a $q_{u} / \sigma_{t}$ value of more than 25 can be obtained. This ratio is usually around 5 for a CPI model (Huang, 1999), which is very low compare to those for rocks. Therefore, a SOCPI model is capable of simulating realistic compressive to tensile strength ratio.

\section{Calibration Procedures}

To examine the usefulness of the calibration charts, an effort was made to develop a synthetic material similar to Pennsylvania Blue Sandstone. The mechanical properties 


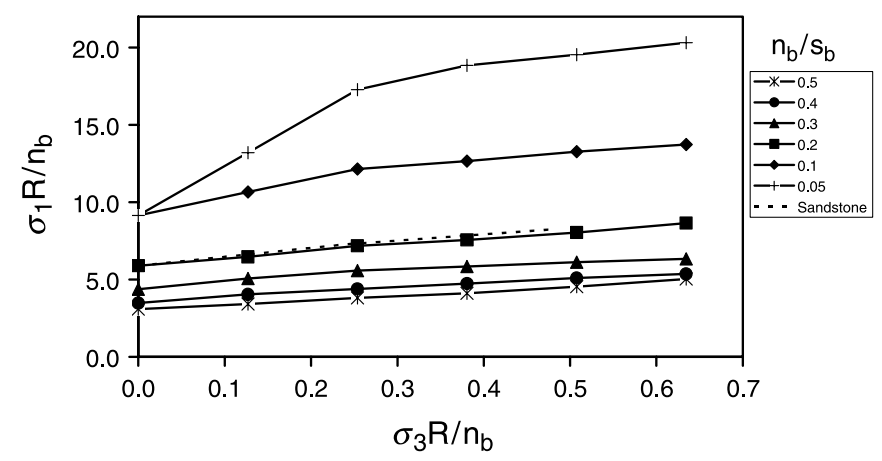

Fig. 11. Normalized failure envelopes for $\sigma_{0} / k_{n}=0.08$ together with the normalized failure envelope of Pennsylvania Blue Sandstone

of this rock were obtained by performing laboratory uniaxial, triaxial and Brazilian tests. This sandstone has a Young's modulus (E) of $27 \mathrm{GPa}$, a Poisson's ratio $(\nu)$ of 0.15 , a tensile strength $\left(\sigma_{t}\right)$ of $9.9 \mathrm{MPa}$, and an unconfined compressive strength $\left(q_{u}\right)$ of $122 \mathrm{MPa}$. Four steps were employed to calibrate a synthetic material similar to the sandstone as explained in the following:

a) The first step was to search among dimensionless or normalized failure envelopes that are shown in Fig. 5. In Fig. 11, the normalized failure envelope of the sandstone together with those of synthetic materials for $\sigma_{0} / k_{n}=0.08$ is shown. This figure confirmed that a curve with $n_{b} / s_{b}=0.2$ had a good match with the failure envelope of sandstone. Hence, $\sigma_{0} / k_{n}=0.08$ and $n_{b} / s_{b}=0.2$ were selected for the synthetic material.

b) Equations $3 \mathrm{a}$ and $3 \mathrm{~b}$ together with $\mathrm{E}=27 \mathrm{GPa}$ and $\nu=0.15$ were used to obtain the apparent Young's modulus and Poisson's ratio of the sandstone as: $\mathrm{E}^{\prime}=28 \mathrm{GPa}$ and $\nu^{\prime}=0.18$. With $\nu^{\prime}=0.18$ and $\sigma_{0} / k_{n}=0.08, k_{s} / k_{n}=0.42$ was found from Fig. 2b. Next, $\mathrm{E}^{\prime} / k_{n}=0.66$ was acquired from Fig. 2a, with $k_{s} / k_{n}=0.42$ and $\sigma_{0} / k_{n}=0.08$. Therefore, the values of $k_{n}=42.4 \mathrm{GPa}$ and $\mathrm{k}_{\mathrm{s}}=17.8 \mathrm{GPa}$ were obtained. Finally, by considering $\sigma_{0} / k_{n}=0.08$ and $k_{n}=42.4 \mathrm{GPa}$, a genesis pressure of $3.4 \mathrm{GPa}$ was calculated.

c) Laboratory experiments showed that the ratio of unconfined compressive strength to tensile strength of sandstone was 12 . This value together with $n_{b} / s_{b}=0.2$, $\sigma_{0} / k_{n}=0.08$, and $k_{s} / k_{n}=0.42$ were used in Fig. $10 \mathrm{~b}$ to obtain an $R / D$ equal to 0.013 . With a specimen width of $D=40 \mathrm{~mm}$ and $R / D=0.013$, the average cylinders radius of $0.52 \mathrm{~mm}$ was calculated.

d) The last step was to acquire the normal and shear bonds of the synthetic material. An approximate value of $R / n_{b}$ was obtained through step (a), when the failure envelope was selected. In fact, $R / n_{b}$ was the scaling factor in plotting the sandstone failure envelope in Fig. 11. A more accurate estimate of $R / n_{b}$ value that incorporates the effect of $k_{s} / k_{n}$ parameter is obtained by using Fig. 3b. With $\sigma_{0} / k_{n}=0.08$, $n_{b} / s_{b}=0.2$ and $k_{s} / k_{n}=0.42$, a $q_{u} R / n_{b}$ equal to 5 was acquired from Fig. $3 \mathrm{~b}$. Inserting $R=0.52 \mathrm{~mm}$ and $q_{u}=122 \mathrm{MPa}$ in the $q_{u} R / n_{b}=5$, a normal bond of $n_{b}=12,664 \mathrm{~N} / \mathrm{m}$ was obtained which together with $n_{b} / s_{b}=0.2$, resulted in a shear 
bond of $s_{b}=63,320 \mathrm{~N} / \mathrm{m}$. Finally, a reasonable friction coefficient of $\mu=0.5$ was assumed for the synthetic material.

In conclusion, the parameters obtained through the above calibration steps were: $\sigma_{0}=3.4 \mathrm{GPa}, \quad k_{n}=42.4 \mathrm{GPa}, \quad k_{s}=17.8 \mathrm{GPa}, \quad n_{b}=12,664 \mathrm{~N} / \mathrm{m}, \quad s_{b}=63,320 \mathrm{~N} / \mathrm{m}$, $R_{\text {ave }}=0.5 \mathrm{~mm}$ and $\mu=0.5$.

\section{Numerical Experiments on the Synthetic Material}

The synthetic material with the microscopic parameters obtained in the previous section should have mechanical properties similar to Pennsylvania Blue Sandstone. To verify that, rectangular synthetic specimens $40 \mathrm{~mm} \times 80 \mathrm{~mm}$ in dimension for biaxial tests and circular specimens $40 \mathrm{~mm}$ in diameter were created using CA2 computer program. The uniaxial test result on the synthetic specimen is demonstrated in Fig. 12 that shows the axial stress-axial strain and axial stress-lateral strain curves. This figure compares well with that of laboratory uniaxial test on the sandstone (Fig. 13). It is interesting to realize that by development of microcracks at an axial

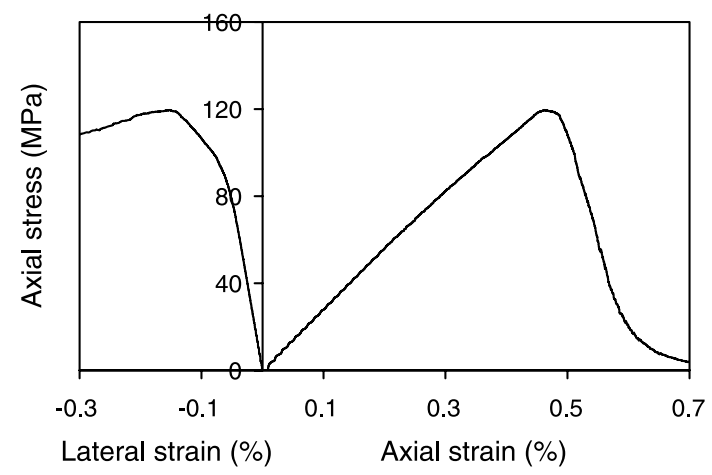

Fig. 12. Axial stress-axial strain and axial stress-lateral strain curves of the synthetic material in a numerical uniaxial test

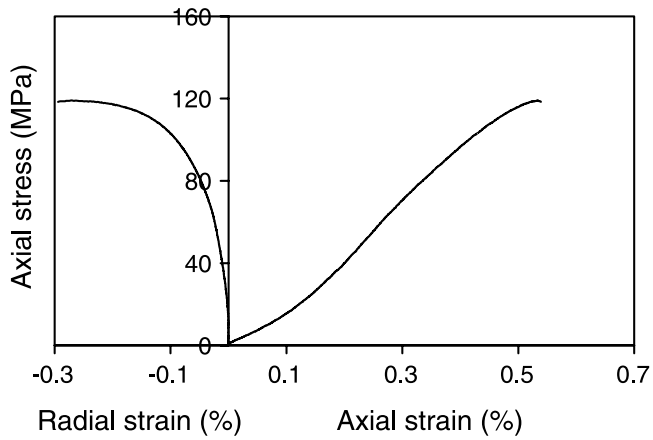

Fig. 13. Axial stress-axial strain and axial stress-lateral strain curves of the sandstone in a physical uniaxial test 


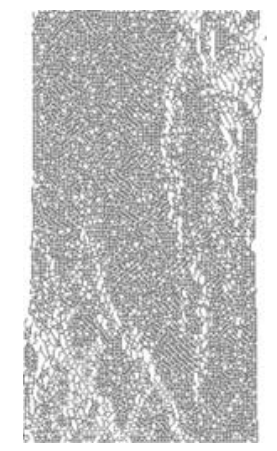

Fig. 14. Damaged specimen in a numerical uniaxial test

stress of about $60 \%$ of unconfined compressive strength, dilatation emerges as is clearly visible in axial stress-lateral strain curves of both physical and numerical specimens. From the initial slopes of the curves in Fig. 12, Young's modulus and Poisson's ratio of the synthetic material was calculated to be $\mathrm{E}=27.2 \mathrm{GPa}$ and $\nu=0.145$ that are in good agreement with those for the sandstone. The damaged numerical specimen at the end of the uniaxial test is shown in Fig. 14. In this figure, damaged contacts of the cylinders are shown with lighter color; the synthetic specimen shows both shear banding and axial splitting at the end of numerical test.

Some numerical biaxial tests with confining pressure of $\sigma_{3}=5,10,15,20 \mathrm{MPa}$ were conducted as well. Figure 15 shows the stress-strain curves for these biaxial tests from which the peak and residual failure envelopes can be deduced. These failure envelopes together with those for the sandstone are shown in Fig. 16 with great agreement. This figure confirms that the curvature of failure envelope has been simulated accurately.

For the numerical Brazilian test, a set up demonstrated in Fig. 7 was employed, and a tensile strength of $\sigma_{t}=9.9 \mathrm{MPa}$ was inferred that is in excellent agreement with that of the physical experiment. The damaged synthetic Brazilian specimen (Fig. 17)

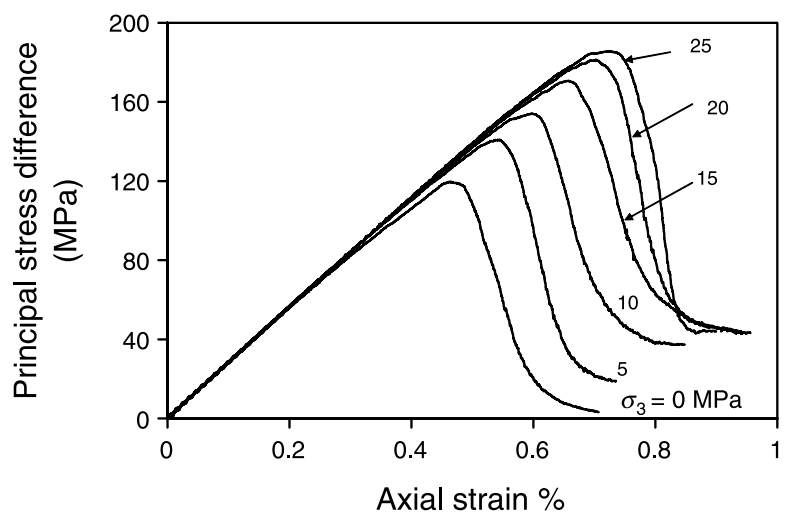

Fig. 15. Principal stress difference $\left(\sigma_{1}-\sigma_{3}\right)$ versus axial strain in numerical biaxial tests with different confining pressures 


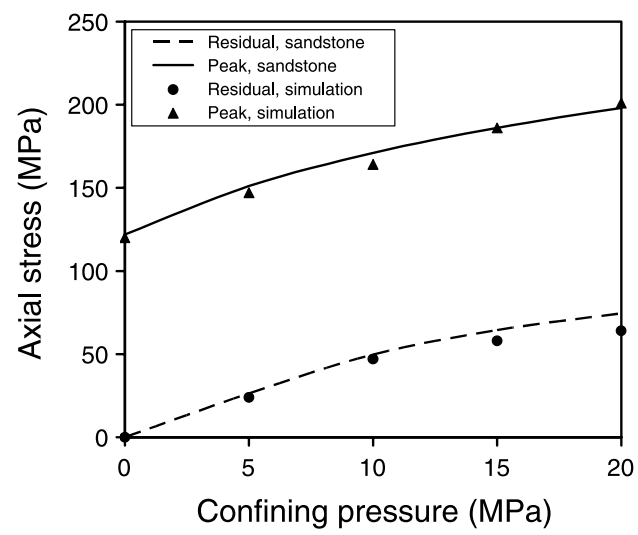

Fig. 16. Peak and residual failure envelopes of the sandstone and the synthetic material

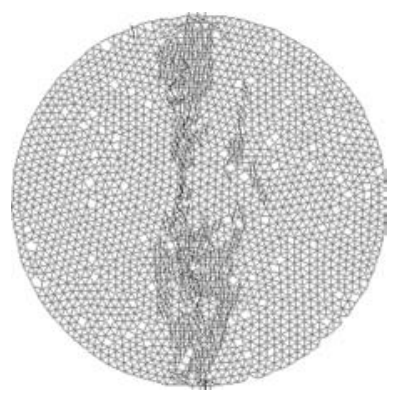

Fig. 17. Developed cracks in the numerical Brazilian test

indicates the development of a macroscopic tensile crack along the vertical diameter that is consistent with physical observation.

In addition to the triaxial and Brazilian laboratory tests, two non-conventional triaxial tests were conducted on the Pennsylvania Blue Sandstone to investigate the effect of two different stress paths on the rock strength. For S2 sample of sandstone, a triaxial test was conducted. The initial confining stress was $25 \mathrm{MPa}$ and subsequently, an axial stress of $175 \mathrm{MPa}$ was applied and kept constant. The confining stress was then reduced gradually until the sample failure. The sample failed at a confining stress of $10 \mathrm{MPa}$. The final state of stress is shown in Fig. 18 together with the failure envelope. In this figure, $p$ and $q$ are defined as:

$$
\begin{aligned}
& p=\left(\sigma_{1}+\sigma_{3}\right) / 2, \\
& q=\left(\sigma_{1}-\sigma_{3}\right) / 2 .
\end{aligned}
$$

For the second physical experiment, sample S5, a confining stress of $10 \mathrm{MPa}$ was applied initially and followed by an axial stress of $150 \mathrm{MPa}$. The confining stress was then increased to $20 \mathrm{MPa}$ and kept constant while the axial stress was increased until the sample failure. The sample failed at an axial stress of $218 \mathrm{MPa}$. From Fig. 18, it is 


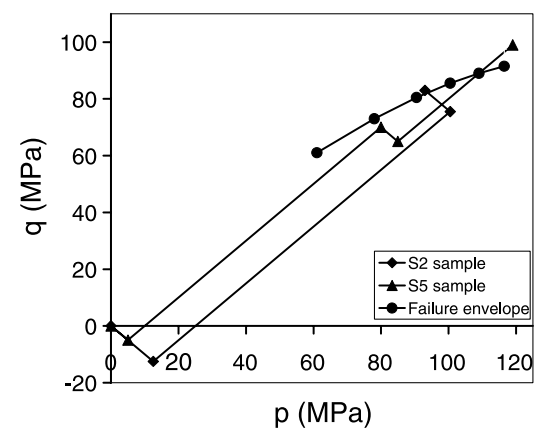

Fig. 18. Stress paths followed for $\mathrm{S} 2$ and $\mathrm{S} 5$ samples of the sandstone

clear that samples S2 and S5 that were subjected to stress paths different from that in a triaxial compression test, show strengths that are relatively consistent with the prediction obtained by the conventional failure criterion.

To approximately simulate these two stress paths, two numerical biaxial tests were conducted. In a biaxial test, the stress path is not the same as that in a triaxial test, but the effect of intermediate principal stress is usually small and it is ignored here. For the synthetic sample S2b, an initial lateral pressure of $25 \mathrm{MPa}$ was followed by an axial stress of $175 \mathrm{MPa}$. Thereafter, the axial stress was kept constant while the lateral pressure was gradually reduced until sample failure. For the synthetic sample S5b, a confining pressure of $10 \mathrm{MPa}$ was initially applied and followed by an axial stress of $150 \mathrm{MPa}$. The confining pressure was then increased to $20 \mathrm{MPa}$ and kept constant while the axial stress gradually increased until the sample failure. The stress paths and failure points are shown in Fig. 19 together with the failure envelope of the synthetic material. It is clear that the failure points are in close agreement with the convential failure envelope. Besides, comparison of Figs. 18 and 19 indicates that the calibrated synthetic material has been capable of simulating the failure of sandstone reasonably well, even when some non-conventional stress paths were followed.

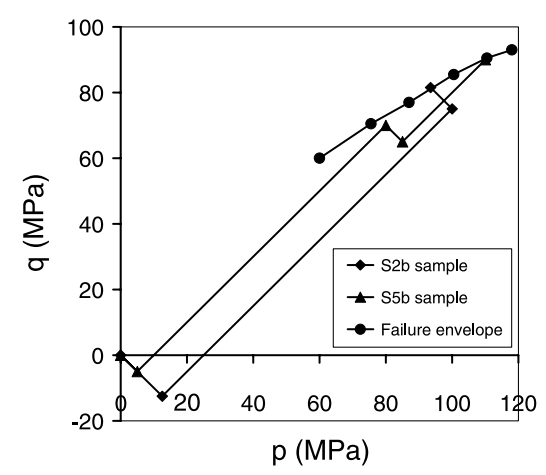

Fig. 19. Stress paths followed for $S 2 b$ and $S 5 b$ samples of the synthetic material 


\section{Conclusion}

Dimensional analysis was employed to establish a fast and efficient tool for calibration of synthetic materials made of bonded circular particles. Several dimensionless parameters and graphs were presented. Careful examination of these graphs shows that the applied genesis pressure during sample preparation is an important factor in dictating the deformation and strength properties of a synthetic material. By using high genesis pressure that induces small overlap of circular cylinders, more realistic friction angle, failure envelope and tensile strength can be simulated that was not possible previously by using circular cylinders with zero genesis pressure.

A synthetic material similar to Pennsylvania Blue Sandstone was developed using the proposed systematic approach for sample calibration. Numerical experiments showed that this model could mimic some of the deformation and failure characteristics of the sandstone closely. For example, the peak and residual failure envelopes and their curvature were simulated accurately. In addition, two non-conventional stress paths were applied to both physical and numerical specimens that showed results in close agreement. In summary, it was shown that the numerical model together with the calibration charts offer a robust and useful tool in simulating many aspects of rock mechanical behavior.

\section{References}

Bazant, Z. P., Pijaudier-Cabot, G. (1988): Nonlocal continuum damage, localization instability and convergence. J. Appl. Mech. 55, 287-293.

Bazant, Z. P., Planas, J. (1998): Fracture and size effect in concrete and other quasibrittle materials. CRC Press, Boca Raton.

Carol, I., Bazant, Z., Prat, P. (1991): Geometric damage tensor based on microplane model. J. Engng. Mech. 117(10), 2429-2448.

Cundall, P., Strack, O. D. L. (1979): A discrete element model for granular assemblies. Geotechnique 29, 47-65.

De Borst, R. (2002): Fracture in quasi-brittle materials: a review of continuum damage-based approaches. Engng. Fract. Mech. 69, 95-112.

De Borst, R., Muhlhaus, H. B. (1991): Continuum models for discontinuum media. Fracture process in concrete, rock and ceramics. In: Van Mier, J. G. M., Rots, J. G., Bakker, A. (eds) Proc. International RILEM/ESIS Conference, Vol. 2, Macroscopic modeling and structural Engineering, E \& FN Spon, London.

De Borst, R., Muhlhaus, H. B. (1992): Gradient-dependent plasticity: formulation and algorithmic aspects. Int. J. Numer. Methods Engng. 35, 521-539.

Fakhimi, A. (1998): Theory and user manual of CA2 computer program. Report no. 262 (in Farsi), Building and Housing Research Center, Tehran, Iran.

Fakhimi, A. (2004): Application of slightly overlapped circular particles assembly in numerical simulation of rocks with high friction angles. Engng. Geology 74, 129-138.

Goodman, R. E. (1989): Introduction to rock mechanics, 2nd edn. John Wiley \& Sons, Chichester.

Huang, H. (1999): Discrete element modeling of tool-rock interaction. PhD dissertation, Department of Civil Engineering, University of Minnesota, USA.

Itasca Consulting Group (1999): Particle flow codes in 2 or 3 dimension $\left(\mathrm{PFC}^{2 \mathrm{~d}} \& \mathrm{PFC}^{3 \mathrm{~d}}\right.$ ), Minneapolis, MN, USA. 
Pande, G. N., Xiong, W. (1982): An improved multi-laminate model of jointed rock masses. International Symposium on Numerical Models in Geomechanics. Zurich, 13-17.

Potyondy, D., Cudall, P. (2001): The PFC model for rock: predicting rock mass damage at the underground research laboratory. Report no. 06819-REP-01200-10061-R00, Itasca Consulting Group, MN, USA.

Rudnicki, J. W., Rice, J. R. (1975): Conditions of the localization of deformation in pressuresensitive materials. J. Mech. Phys. Solids, 23, 371-394.

Sedov, L. I. (1993): Similarity and dimensional methods in mechanics, $10^{\text {th }}$ edn. CRC Press, Boca Raton.

Steen, V. D., Vervoort, A., Napier, J. A. L., Durrheim, R. J. (2003): Implementation of a flaw model to the fracturing around a vertical shaft. Rock Mech. Rock Engng. 36(2), 143-161.

Ting, J. M., Khwaja, M., Meachum, L. R., Rowell, J. D. (1993): An ellipse-based discrete element model for granular materials. Int. J. Numer. Anal. Methods Geomech. 17, 603-623.

Vardoulakis, I., Sulem, J. (1995): Bifurcation analysis in geomechanics. Blackie Academic and Professional, Glasgow.

Vermeer, P. A., De Borst, R. (1984): Non-associated plasticity for soil, concrete and rock. Heron 29(3), 1-65.

Villegas, T. (2004): Calibration of a discrete element model for rock: a dimensional analysis approach. MS dissertation, Department of Mineral Engineering, New Mexico Tech, Socorro, NM, USA.

Author's address: Dr. Ahmadali Fakhimi, Department of Mineral Engineering, New Mexico Tech, 801 Leroy Pl, Socorro, NM 87801, USA; e-mail: hamed@nmt.edu 